\title{
Right diaphragmatic eventration associated with dextrocardia, hypoplasia of the right lower pulmonary lobe, and agenesis of the inferior vena cava
}

El Hassane Kabiri, MD, Abdelfettah Zidane, MD, Adil Arsalane, MD, and Fouad Atoini, MD, Rabat, Morocco

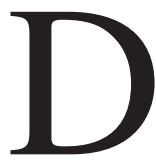
iaphragmatic eventration (DE) is defined as the abnormal and permanent elevation of the hemidiaphragm without defects. It may be congenital (muscular hypoplasia) or acquired (injury of the phrenic nerve).

DE has been reported alone or in association with other malformations. We report a 39-year-old woman with right-sided DE associated with dextrocardia, hypoplastic right lower lobe of the lung, and malformation of the azygos venous systems with agenesis of the inferior vena cava.

\section{Clinical Summary}

A 39-year-old woman was referred to our department for dyspnea, chest pain, recurrent pulmonary infection (right lower lobe), and a recent episode of vomiting. Chest radiography (Figure 1) showed an elevation of the posterior portion of the right hemidiaphragm and dextrocardia. A posterolateral thoracotomy was performed. After pulmonary liberation, we noticed an elevation of the posterior portion of the diaphragm (the anterior portion was normal), hypoplasia of the lower pulmonary lobe with a small artery, and an inferior pulmonary vein associated with a large azygos vein $(\sim 20$ $\mathrm{mm}$ in diameter) from the diaphragm to the junction with the superior vena cava. We performed a right lower lobectomy and a surgical plicature of the posterior portion of the diaphragm with nonabsorbable suture. This suture was reinforced with a synthetic prosthesis. A thoracic drain was left in place for 4 days. The postoperative course was uneventful. Histopathologic examination of the lower lobe revealed a fibroinflammatory tissue with pulmonary parenchyma destruction. Computed tomography (Figure 2) was performed at D10 to evaluate the venous system malformation; this showed an agenesis of the inferior vena cava with dilatation of the azygos and hemiazygos venous systems.

\footnotetext{
From the Department of Thoracic Surgery, Mohamed V Military Teaching Hospital (Hôpital Militaire d'Instruction Mohammed V), Rabat, Morocco. Received for publication Nov 16, 2006; accepted for publication Dec 12 , 2006.

Address for reprints: El Hassane Kabiri, MD Immeuble 29 Appt 7, Résidence Mes Ellil, Secteur 23, Riad 10100, Rabat, Morocco (E-mail: hassankabiri@yahoo.com)

J Thorac Cardiovasc Surg 2007;133:1380-1

$0022-5223 / \$ 32.00$

Copyright (C) 2007 by The American Association for Thoracic Surgery doi:10.1016/j.jtcvs.2006.12.054
}

\section{Discussion}

DE is rare and represents atrophy and distension of the dia-

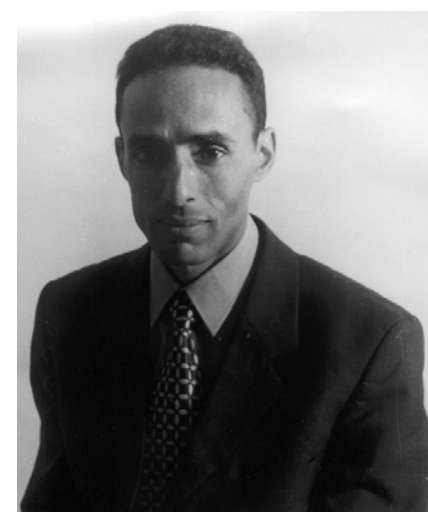

Dr Kabiri phragmatic muscle. The incidence of DE is difficult to estimate because of its rarity, and the majority of cases are asymptomatic. DE may be congenital or acquired, complete or partial, unilateral or bilateral. ${ }^{1} \mathrm{DE}$ has been reported in the literature in association with other anomalies, such as bronchopulmonary foregut malformations ${ }^{2}$ (congenital cystic adenomatoid malformation, congenital lobar emphysema, sequestration, bronchogenic cyst, tracheal or bronchial atresia); cardiac malformation (dextrocardia); and gastrointestinal $^{3}$ (hiatal hernia, esophageal duplication cysts), renal, and musculoskeletal anomalies. These recognized or unrecognized patterns of malformation were reported in 3\% to $40 \%$ of cases. ${ }^{3}$ The coexistence of these anomalies attests to the common embryologic origin of these lesions, ${ }^{4}$ which have a serious effect on the outcome. The association with agenesis of the inferior vena cava and dilatation of the azygos and hemiazygos venous systems has not been reported. Dyspnea is the most frequent symptom, typically worsened by the prone position; other clinical symptoms include chest pain, recurrent pulmonary infections, vomiting, and/or cardiac manifestations. Chest radiography usually permits the diagnosis of DE with visualization of the intact dome of the diaphragm, but it is difficult to distinguish from other diagnoses, such as pleural effusion; diaphragmatic hernia; and pulmonary, hepatic, or spleen involvement. Other imaging investigations should be performed, particularly thoracoabdominal computed tomography scans or magnetic resonance imaging, to exclude other

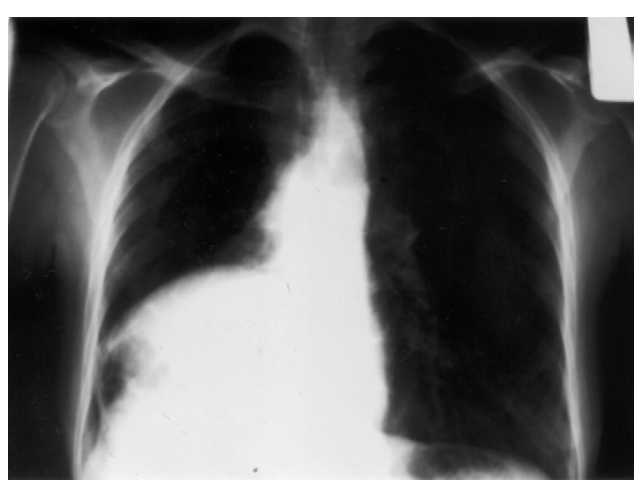

Figure 1. Chest radiograph indicating an elevation of the right hemidiaphragm with dextrocardia. 


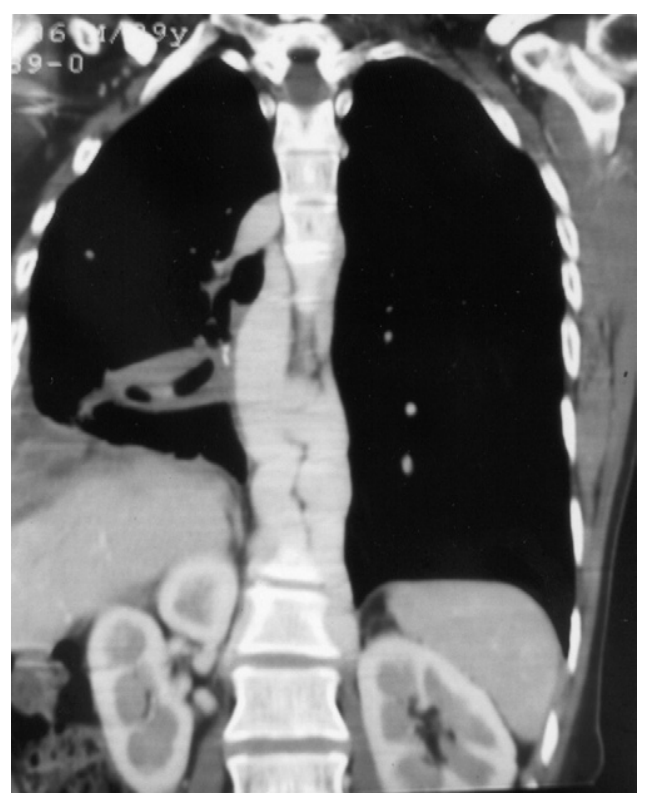

Figure 2. Computed tomography confirmed the dilatation of the azygos venous systems with agenesis of the inferior vena cava.

pathologic conditions, reach the other associated malformations, and determine the consequences of this anomaly. ${ }^{2,3}$

Immediate surgical treatment of all congenital DE is necessary because of the risk of complications (pulmonary destruction).
Surgical treatment of DE through a thoracotomy is the procedure of choice. Various techniques have been proposed. Diaphragmatic plication is usually sufficient, but when the diaphragm is too fragile, a synthetic prosthesis can be added. Some authors recommend the excision of the central thin portion in congenital forms to bring tendinomuscular tissues together and suture. Plication of the diaphragm through a standard thoracotomy is the most frequently used approach; it carries a low morbidity and no mortality.

Diaphragmatic plication through thoracoscopy or videoassisted thoracoscopic surgery recently became an alternative technique to conventional thoracotomy, with a lower rate of complications and shorter hospital stay.

The diverse and serious problems caused by associated congenital malformations must be diagnosed and treated immediately to prevent complications.

We thank Dominique Gossot, MD, Institut Mutualiste Montsouris, Paris, France, for help in editing this article.

\section{References}

1. Tiryaki T, Livanelioglu Z, Atayurt H. Eventration of the diaphragm. Asian J Surg. 2006;29:8-10.

2. Rajev C, Dharmendra S, Paras K, Siddharth S, Roy CS, Manjula J. Congenital cystic adenomatoid malformation with ipsilateral eventration of the diaphragm. Indian J Pediatr. 2006;73:832-4.

3. Al-Afraj AA, Chirurgie AF, Khwaja MS, Mitra DK. Congenital diaphragmatic eventration associated with massive hiatal hernia. Indian J Pediatr. 2005;72:181.

4. Singal AK, Srinivas M, Bhatnagar V. Bronchopulmonary foregut malformation in association with diaphragmatic eventration. J Pediatr Surg. 2006;41:1329-31.

5. Shin JS. Plication for diaphragmatic eventration. A simple technique, not a simple problem. Chest. 2004;125:1170-1. 